\title{
Invasive Papillary Carcinoma of the Breast: Clinicopathological Features and Hormone Receptor Profile
}

Atif A. Hashmi ${ }^{1}$, Shahzeb Munawar ${ }^{2}$, Naumana Rehman ${ }^{3}$, Omer Ahmed ${ }^{4}$, Sabeeh Islam ${ }^{5,} 6$, Ishaq Azeem Asghar ${ }^{7}$, Anoshia Afzal ${ }^{8}$, Muhammad Irfan ${ }^{9}$, Farozaan Shamail ${ }^{10}$, Syed J. Ali ${ }^{11}$

1. Pathology, Liaquat National Hospital and Medical College, Karachi, PAK 2. Internal Medicine, Liaquat College of Medicine and Dentistry, Karachi, PAK 3. Pathology, Khyber Medical University, Peshawar, PAK 4. Internal Medicine, Liaquat National Hospital and Medical College, Karachi, PAK 5. Internal Medicine, St. Vincent Health Center, Buffalo, USA 6. Internal Medicine, Faisalabad Medical University, Faisalabad, PAK 7. Pathology, Ascension St. John Hospital, Detroit, USA 8. Pathology, University of Oklahoma Health Sciences Center, Oklahoma City, USA 9. Statistics, Liaquat National Hospital and Medical College, Karachi, PAK 10. Pathology, Ziauddin University, Karachi, PAK 11. Pathology, Dow University of Health Sciences, Karachi, PAK

Corresponding author: Atif A. Hashmi, atifhashmi345@gmail.com

\section{Abstract}

\section{Introduction}

Papillary neoplasms are a heterogeneous group of breast lesions, ranging from benign to in situ and invasive malignant tumors. The term invasive papillary carcinoma (IPC) is reserved for rare invasive breast tumors showing greater than $90 \%$ papillary morphology. The clinical, epidemiological and pathological characteristics of IPC are not widely described in the existing literature; therefore, in this study, we evaluated the clinicopathological features and biomarker profile of IPC and compared it with invasive ductal carcinoma (IDC) diagnosed in the same study duration.

\section{Methods}

A retrospective study was conducted in the Department of Histopathology, Liaquat National Hospital and Medical College, from January 2013 to December 2020. During the study period, 44 cases of IPC and 1,268 cases of IDC were diagnosed. Slides and blocks of all cases were retrieved and histopathological diagnosis was reviewed. Estrogen receptor (ER), progesterone receptor (PR), human epidermal growth factor receptor 2 (HER2/neu), and Ki67 immunohistochemical (IHC) stains were applied on representative tissue blocks.

\section{Results}

The mean age of the patients with IPC was $58.77 \pm 8.38$ years, and the mean Ki67 index was $19.95 \pm 21.12 \%$. The mean tumor size was $32.41 \pm 17.39 \mathrm{~mm}$, and most tumors (59.1\%) were tumor (T)-stage T2. Axillary metastasis was present in $13.6 \%$ cases, and $86.4 \%$ cases had nodal (N)-stage N0. ER and PR expression was noted in $72.7 \%$ cases, and HER2/neu positivity was seen in $13.6 \%$ cases. IPC cases had a higher mean age than IDC. Conversely, IPC had a lower mean Ki67 index than IDC. Similarly, IPC cases were found to have a lower frequency of axillary metastasis than IDC. IPC was noted to have a lower frequency of T3-stage and lymphovascular invasion than IDC. A higher expression of PR and lower frequency of HER2/neu expression was noted in IPC than IDC.

\section{Conclusion}

IPC is a rare malignant papillary breast tumor with a wide differential diagnosis and therefore poses a significant diagnostic challenge. We found that IPC had a favorable pathological profile than IDC, in terms of T-stage, Ki67 index, axillary metastasis, PR and HER2/neu expression.

Categories: Pathology, General Surgery, Oncology

Keywords: invasive papillary carcinoma, invasive ductal carcinoma, breast cancer, estrogen receptor, progesterone receptor, human epidermal growth factor receptor 2, ki67, papillary lesions

\section{Introduction}

Papillary neoplasms are a heterogeneous group of breast lesions, ranging from benign to in situ and invasive malignant tumors [1,2]. Owing to growth inside the ducts, most of the malignant papillary breast tumors are in situ, including encapsulated and solid papillary carcinoma [3,4]. The term invasive papillary carcinoma (IPC) is reserved for rare invasive breast tumors showing greater than $90 \%$ papillary morphology. Applying the above-mentioned morphological criteria, the incidence of IPC is rare ( $0.5 \%$ of all breast cancers) [5]. It is noteworthy that invasive carcinoma arising in the background of solid and encapsulated papillary carcinoma is not considered IPC. Moreover, invasive micropapillary carcinoma (IMPC) is also excluded from the 


\section{Cureus}

category of IPC, as IMPC is characterized by nests of tumor cells with reverse polarization, present in cleftlike spaces. IMPC is associated with frequent lymphovascular invasion and poor prognosis [6]. Furthermore, the diagnostic consideration includes metastasis from other sites, notably, lung and female genital tract, especially, ovary. The clinical, epidemiological and pathological characteristics of IPC are not widely described in the existing literature; therefore, in this study, we evaluated the clinicopathological features and biomarker profile of IPC and compared it with invasive ductal carcinoma (IDC) diagnosed in the same study duration.

\section{Materials And Methods}

A retrospective study was conducted in the Department of Histopathology, Liaquat National Hospital and Medical College, from January 2013 to December 2020. Cases with a biopsy-proven diagnosis of primary breast cancer, undergoing excision were included in the study. Cases with metastasis or those who received neo-adjuvant chemoradiation before surgery were excluded from the study. Specimens included modified radical mastectomy (MRM), simple mastectomy with sentinel lymph node sampling (intraoperatively), and wide local excision with/without axillary dissection. All specimens were received in the histopathology laboratory and were grossly examined, followed by gross dissection according to standard protocols. Representative sections were taken from tumor, resection margins, skin (for mastectomies), non-neoplastic breast tissue, and axillary lymph nodes. During the study period, 44 cases of IPC and 1,268 cases of IDC were diagnosed. Slides and blocks of all cases were retrieved and histopathological diagnosis was reviewed. Estrogen receptor (ER), progesterone receptor (PR), human epidermal growth factor receptor 2 (HER2/neu), and Ki67 immunohistochemical (IHC) stains were applied on representative tissue blocks. More than $1 \%$ expression ER and PR was taken as positive. Nuclear staining for Ki67 was interpreted in the hot spots (area of the tumor with maximum staining) and reported as an average percentage of staining. For HER2neu IHC interpretation, complete strong staining in more than $10 \%$ of tumor cells was taken as positive (3+). An equivocal (2+) HER2/neu IHC result was further tested by fluorescence in situ hybridization (FISH) study for HER2/neu gene amplification.

Data analysis was performed using Statistical Package for Social Sciences (Version 26.0, IBM Inc., Armonk, USA). Independent t-test, Chi-square, and Fisher's exact tests were used to check the association. P-values < 0.05 were considered significant.

\section{Results}

The mean age of the patients with IPC was $58.77 \pm 8.38$ years, and most of the patients were above 50 years of age. The mean Ki67 index was $19.95 \pm 21.12 \%$, and most patients (59.1\%) had Ki67 index less than $15 \%$. The mean tumor size was $32.41 \pm 17.39 \mathrm{~mm}$, and most tumors (59.1\%) were tumor (T)-stage T2. Axillary metastasis was present in $13.6 \%$ cases, and $86.4 \%$ cases had nodal $(\mathrm{N})$-stage N0. Most of the tumors were grade II (59.1\%). ER and PR expression was noted in $72.7 \%$ cases, and HER2/neu positivity was seen in $13.6 \%$ cases. The lymphovascular and dermal lymphatic invasions were seen in $4.5 \%$ and $9.1 \%$ cases, respectively (Table 1). 


\section{Cureus}

T2, n (\%)

26 (59.1)

T3, n (\%)

$4(9.1)$

Axillary metastasis

Present, n (\%)

6 (13.6)

Absent, n (\%)

38 (86.4)

$\mathrm{N}$-stage, n (\%)

No, n (\%)

38 (86.4)

N1, n (\%)

2 (4.5)

N2, n (\%)

2 (4.5)

N3, n (\%)

2 (4.5)

Tumor grade

Grade I, n (\%)

Grade II, n (\%)

$26(59.1)$

Grade III, n (\%)

14 (31.8)

Laterality

Left, n (\%)

18 (40.9)

Right, n (\%)

$26(59.1)$

Specimen type

Modified radical mastectomy, $\mathrm{n}(\%)$

$14(31.8)$

Simple mastectomy with sentinel lymph node dissection, $n$ (\%)

$12(27.3)$

Wide local excision with/without axillary dissection, $\mathrm{n}(\%)$

$18(40.9)$

ER

Positive, n (\%)

32 (72.7)

Negative, n (\%)

$12(27.3)$

PR

Positive, n (\%)

32 (72.7)

Negative, n (\%)

$12(27.3)$

HER2/neu

Positive, n (\%)

6 (13.6)

Negative, n (\%)

38 (86.4)

Lymphovascular invasion

Present, n (\%)

Absent, n (\%)

42 (95.5)

Dermal lymphatic invasion

Present, n (\%)

$4(9.1)$

Absent, n (\%)

$42(90.9)$

TABLE 1: Clinicopathologic characteristics of invasive papillary carcinoma $(n=44)$

SD, standard deviation; T, tumor; N, nodal; ER, estrogen receptor; PR, progesterone receptor; HER2/neu, human epidermal growth factor receptor 2 


\section{Cureus}

Table 2 compares the pathological characteristics of IPC with IDC. A significant difference in clinicopathological characteristics was noted between IPC and IDC, with respect to age, Ki67 index, T-stage, $\mathrm{N}$-stage, lymphovascular invasion, PR and HER2/neu expression. IPC cases had a higher mean age than IDC. Conversely, IPC had a lower mean Ki67 index than IDC. Similarly, IPC cases were found to have a lower frequency of axillary metastasis than IDC. IPC was noted to have a lower frequency of T3-stage and lymphovascular invasion than IDC. A higher expression of PR and lower frequency of HER2/neu expression were noted in IPC than IDC. Although a higher frequency of IPC showed ER expression (than IDC), the difference was not statistically significant.

\begin{tabular}{|c|c|c|c|}
\hline \multirow{2}{*}{$\begin{array}{l}\text { Clinicopathological characteristics and immunohistochemical } \\
\text { expression }\end{array}$} & \multicolumn{2}{|l|}{ Values } & \multirow[b]{2}{*}{ P-value } \\
\hline & $\begin{array}{l}\text { Invasive ductal carcinoma } \\
(\mathrm{n}=1268)\end{array}$ & $\begin{array}{l}\text { Invasive papillary carcinoma } \\
(\mathrm{n}=44)\end{array}$ & \\
\hline Age $\left(\right.$ years) ${ }^{\star}$, mean $\pm S D$ & $51.95 \pm 12.15$ & $58.77 \pm 8.38$ & $<0.0001^{\star \star \star \star}$ \\
\hline Ki67 index $(\%)^{*}$, mean $\pm S D$ & $30.54 \pm 21.60$ & $19.95 \pm 21.12$ & $0.001^{\star \star \star \star}$ \\
\hline \multicolumn{4}{|l|}{ Ki67 index groups ${ }^{\star \star}$} \\
\hline$<15 \%, \mathrm{n}(\%)$ & $362(28.5)$ & $26(59.1)$ & \multirow{4}{*}{$<0.0001^{\star \star \star \star \star}$} \\
\hline $15 \%-24 \%, n(\%)$ & $286(22.6)$ & $8(18.2)$ & \\
\hline $25 \%-44 \%, \mathrm{n}(\%)$ & $282(22.2)$ & $4(9.1)$ & \\
\hline$>44 \%, \mathrm{n}(\%)$ & $338(26.7)$ & $6(13.6)$ & \\
\hline Tumor size $(\mathrm{mm})^{\star}$, mean \pm SD & $36.11 \pm 14.84$ & $32.41 \pm 17.39$ & 0.708 \\
\hline \multicolumn{4}{|l|}{ T-stage } \\
\hline $\mathrm{T} 1, \mathrm{n}(\%)$ & $166(13.1)$ & $16(36.4)$ & \multirow{3}{*}{$<0.0001^{\star \star \star \star}$} \\
\hline T2, n (\%) & 906 (71.5) & $24(54.5)$ & \\
\hline T3, n (\%) & 196 (15.5) & $4(9.1)$ & \\
\hline \multicolumn{4}{|l|}{ Axillary metastasis ${ }^{\star \star}$} \\
\hline Present, n (\%) & $636(50.2)$ & $6(13.6)$ & \multirow{2}{*}{$<0.0001^{\star \star \star \star \star}$} \\
\hline Absent, n (\%) & $632(49.8)$ & $38(86.4)$ & \\
\hline \multicolumn{4}{|l|}{ N-stage ${ }^{\star \star}$} \\
\hline N0, n (\%) & $640(50.5)$ & $38(86.4)$ & \multirow{4}{*}{$<0.0001^{\star * \star \star}$} \\
\hline $\mathrm{N} 1, \mathrm{n}(\%)$ & $260(20.5)$ & $2(4.5)$ & \\
\hline N2, n (\%) & 170 (13.4) & $2(4.5)$ & \\
\hline N3, n (\%) & $198(15.6)$ & $2(4.5)$ & \\
\hline \multicolumn{4}{|l|}{ Tumor grade ${ }^{\star \star \star}$} \\
\hline Grade I, n (\%) & $106(8.4)$ & $4(9.1)$ & \multirow{3}{*}{0.180} \\
\hline Grade II, n (\%) & $586(46.2)$ & $26(59.1)$ & \\
\hline Grade III, n (\%) & $576(45.4)$ & $14(31.8)$ & \\
\hline \multicolumn{4}{|l|}{ Laterality ${ }^{\star \star}$} \\
\hline Left, n (\%) & $630(49.7)$ & $18(40.9)$ & \multirow{2}{*}{0.252} \\
\hline Right, n (\%) & 638 (50.3) & $26(59.1)$ & \\
\hline \multicolumn{4}{|l|}{$\mathrm{ER}^{\star \star}$} \\
\hline Positive, n (\%) & 798 (62.9) & $32(72.7)$ & \multirow{2}{*}{0.185} \\
\hline Negative, n (\%) & $470(37.1)$ & $12(27.3)$ & \\
\hline
\end{tabular}




\section{Cureus}

\begin{tabular}{|c|c|c|c|}
\hline Positive, n (\%) & $646(50.9)$ & $32(72.7)$ & \multirow{2}{*}{$0.004^{\star \star \star \star}$} \\
\hline Negative, $\mathrm{n}(\%)$ & $622(49.1)$ & $12(27.3)$ & \\
\hline \multicolumn{4}{|l|}{ HER2/neu* } \\
\hline Positive, n (\%) & $446(35.2)$ & $6(13.6)$ & \multirow{2}{*}{$0.003^{\star \star \star \star}$} \\
\hline Negative, n (\%) & $822(64.8)$ & $38(86.4)$ & \\
\hline \multicolumn{4}{|c|}{ Lymphovascular invasion ${ }^{\star \star}$} \\
\hline Present, n (\%) & $314(24.8)$ & $2(4.5)$ & \multirow{2}{*}{$0.002^{\star \star \star \star}$} \\
\hline Absent, n (\%) & 954 (75.2) & $42(95.5)$ & \\
\hline \multicolumn{4}{|c|}{ Dermal lymphatic invasion** } \\
\hline Present, $n(\%)$ & $156(12.3)$ & $4(9.1)$ & \multirow{2}{*}{0.522} \\
\hline Absent, n (\%) & $1112(87.7)$ & $40(90.9)$ & \\
\hline
\end{tabular}

\section{TABLE 2: Comparison of clinicopathologic characteristics of invasive papillary carcinoma with}

invasive ductal carcinoma of breast

${ }^{*}$ Independent t-test was applied, ${ }^{\star \star}$ Chi-square test was applied, ${ }^{\star \star \star}$ Fisher's exact test was applied, ${ }^{\star \star \star \star} p$-value significant as $<0.05$

SD, standard deviation; T, tumor; N, nodal; ER, estrogen receptor; PR, progesterone receptor; HER2/neu, human epidermal growth factor receptor 2

\section{Discussion}

We found a low frequency of IPC in our population. Conversely, IPC cases were noted to have a better pathological profile, in terms of prognostic features, such as lower Ki67 index, T-stage and N-stage than IDC. Similarly, a higher frequency of PR and lower frequency of HER2/neu expression portend a better biomarker profile in IPC.

Papillary lesions of the breast encompass a range of benign and malignant lesions, ranging from intraductal papilloma, atypical hyperplasia involving a ductal papilloma, as well as ductal carcinoma in situ, solid papillary carcinoma, encapsulated papillary carcinoma and IPC [1,2]. The incidence of IPC is low compared to IDC [5], and the prognosis is better than other malignant entities of the breast $[2,5]$.

The differential diagnoses include solid papillary carcinoma, encapsulated (intra-cystic) papillary carcinoma, IMPC and metastatic papillary carcinoma [2,5]. The typical age at presentation of IPC ranges from 63 to 67 years and presenting complaints include bloody discharge from the nipple, an abnormal mass, and radiographic abnormalities. IPC can arise in a preexisting papilloma, but it is not always the case. Metastasis is rare in IPC. IPC is usually positive for ER and gross cystic disease fluid protein-15 (GCDFP-15) with variable-positive staining for synaptophysin and neuron-specific enolase. Myoepithelial markers such as calponin, smooth muscle myosin heavy chain, and tumor protein-63 (p63) are negative in the peripheral cells and the cells surrounding papillary cores $[1,2,7]$.

Vural et al. analyzed data of 24 cases of IPC and found that IPC had an overall better survival and prognosis than other breast cancers [8]. IPCs are usually unilateral but can have a bilateral presentation rarely [9].

We acknowledge a few limitations of our study. Most importantly, clinical follow-up data were not available to compare the difference in cancer-specific and overall survival between IPC and IDC. Moreover, the number of cases of IPC was low.

\section{Conclusions}

IPC is a rare variant of invasive breast carcinoma. It is important to distinguish IPC from in situ variants of papillary carcinoma, as well as IDC. We found a prognostically better pathological and biomarker profile of IPC than IDC. Apart from the lower frequency of lymphovascular invasion and axillary metastasis, and lower T-stage and Ki67 index, IPC also had a higher frequency of PR and lower frequency of HER2/neu expression. All these features confer a favorable prognostic and biomarker profile of IPC; however, large-scale studies are needed to compare survival differences between IPC and IDC.

\section{Additional Information}




\section{Disclosures}

Human subjects: Consent was obtained or waived by all participants in this study. N/A issued approval N/A. N/A. Animal subjects: All authors have confirmed that this study did not involve animal subjects or tissue. Conflicts of interest: In compliance with the ICMJE uniform disclosure form, all authors declare the following: Payment/services info: All authors have declared that no financial support was received from any organization for the submitted work. Financial relationships: All authors have declared that they have no financial relationships at present or within the previous three years with any organizations that might have an interest in the submitted work. Other relationships: All authors have declared that there are no other relationships or activities that could appear to have influenced the submitted work.

\section{References}

1. Hashmi AA, Faraz M, Rafique S, Adil H, Imran A: Spectrum of papillary breast lesions according to World Health Organization Classification of Papillary Neoplasms of Breast. Cureus. 2020, 12:e11026. 10.7759/cureus.11026

2. Wei S: Papillary lesions of the breast: an update . Arch Pathol Lab Med. 2016, 140:628-43. 10.5858/arpa.2015-0092-RA

3. Hashmi AA, Iftikhar SN, Haider R, Haider R, Irfan M, Ali J: Solid papillary carcinoma of breast: clinicopathologic comparison with conventional ductal carcinoma of breast. Cureus. 2020, 12:e11172. 10.7759/cureus.11172

4. Hashmi AA, Iftikhar SN, Munawar S, Shah A, Irfan M, Ali J: Encapsulated papillary carcinoma of breast: clinicopathological features and prognostic parameters. Cureus. 2020, 12:e11282. 10.7759/cureus.11282

5. Louwman MW, Vriezen M, van Beek MW, et al.: Uncommon breast tumors in perspective: incidence, treatment and survival in the Netherlands. Int J Cancer. 2007, 121:127-35. 10.1002/ijc.22625

6. Hashmi AA, Aijaz S, Mahboob R, et al.: Clinicopathologic features of invasive metaplastic and micropapillary breast carcinoma: comparison with invasive ductal carcinoma of breast. BMC Res Notes. 2018, 11:531. 10.1186/s13104-018-3623-Z

7. Pal SK, Lau SK, Kruper L, et al.: Papillary carcinoma of the breast: an overview . Breast Cancer Res Treat. 2010, 122:637-45. 10.1007/s10549-010-0961-5

8. Vural O, Alnak A, Altundag K: Invasive papillary carcinoma of the breast: an overview of twenty-four cases . Am Surg. 2012, 78:144-5. 10.1177/000313481207800311

9. Elverici E, Barça AN, Türksoy O, Araz L, Yüksel E: Bilateral invasive papillary carcinoma of the breast . Clin Imaging. 2007, 31:419-21. 10.1016/j.clinimag.2007.02.029 